\title{
Wikimedica: une Plateforme Collaborative de Transfert des Connaissances Médicales en Libre Accès
}

\author{
Antoine Mercier-Linteau ${ }^{1}$ \\ ${ }^{1}$ Vice-président de Wikimedica, Faculté of Médecine, Université Laval, Québec, Canada
}

RÉSUMÉ

La médecine moderne ne peut plus se pratiquer sans l'aide de bases de connaissances cliniques. Or, toutes celles disponibles sont payantes et fermées et s'il est un domaine qui pourrait bénéficier d'un accès libre à de l'information fiable, de qualité et à jour, c'est celui de la santé. Wikimedica (http://wikimedi.ca) est une plateforme libre accès conçue à cette fin qui permet tant aux cliniciens de terrain qu'aux étudiants de collaborer dans la création et l'amélioration des connaissances essentielles à leur professions.

ABSTRACT

Modern medicine cannot be practiced without the help of basic clinical knowledge. However, all that is available is payment based and restricted, and if ever there was a field in which one could benefit from free-access to trustworthy, high quality and up to date information, it would be healthcare. Wikimedica (http://wikimedi.ca) is a free-access platform created for this purpose which allows working clinicians and students to collaborate on the creation and the improvement of the knowledge essential to their professions.

V oilà bien longtemps que l'étendue de la connaissance médicale a outrepassé les limites d'un seul cerveau et il est désormais impossible de pratiquer la médecine sans s'aider de bases de connaissances cliniques. Que ce soit pour confirmer un diagnostic ou choisir un traitement, elles sont devenues indispensables lorsque vient le temps d'orienter nos décisions de soins. Dans le domaine, les options se limitent essentiellement à UpToDate ou Dynamed; tous deux demandant le gros prix pour l'accès à leur service, surtout pour les institutions. À cet effet, plusieurs d'entre elles ont été contraintes, devant une augmentation des frais d'accès dépassant le $600 \%$ en 8 ans, à délaisser UpToDate pour son seul compétiteur (1).

Pourtant, s'il est un domaine où l'accès à la connaissance devrait être libre (consultation et réutilisation sans barrière légale, monétaire ou technique), c'est bien celui de la santé : nos systèmes publics en sont tributaires, sa production a largement été financée par nos impôts et la qualité des soins est somme toute garante d'une information de qualité et à jour. C'est un impératif moral. Alors pourquoi n'existe-t-il pas de base de connaissances cliniques complète, fiable et en libre accès? Le célèbre Wikipédia est largement utilisé à ces fins mais tant son mandat encyclopédique que son manque de complétude sur certains sujets de pointe rendent son utilité limitée (2). Citons aussi WikEM, une très bonne ressource elle aussi en libre accès, mais limitée à la médecine d'urgence. Le Dr. James Heilman (urgentologue britano-colombien et ardent défenseur du libre accès), dans un article intitulé Open Access to a High-Quality, Impartial, Point-of-Care Medical Summary Would Save Lives: Why Does It Not Exist? (3), explore les raisons pour lesquelles le libre accès n'a pas percé en clinique. Quelques initiatives se sont attaquées à ce défi de taille dans le passé, comme Medpedia ou Wikemerg.ca, mais quasiment toutes ont échoué, n'ayant pu trouver d'adéquation pour ses éditeurs entre compensation monétaire, prestige et avancement de carrière (3). À ceci j'ajouterai que l'échec de ces projets est également attribuable à leur utilisation d'un modèle éditorial classique par comité exclusif d'experts. II est également possible que la communauté médicale n'était tout simplement par mûre pour opérer une transition vers le libre accès. La seule initiative ayant eu un semblant de succès est le contenu médical sur Wikipédia (qui ironiquement ne cible pas les professionnels) ce qui s'explique principalement de par son processus éditorial collaboratif, informel et révisé par les pairs. Dr. Heilman suggère ensuite que si les créateurs de ce contenu sur Wikipédia, au nombre de 2500, choisissaient d'écrire quelques articles de niveau clinique, d'adapter ceux existants ou d'en réviser d'autres, en peu de temps nous aurions bâti cette base de connaissance impartiale, de qualité et surtout, en libre accès. Bref, la force du nombre...Et en santé, cette

Keywords: Wiki; Collaboration; Knowledge Transfer; Inter-professionnal; Open-access 
force existe, il suffit seulement de la canaliser. Chaque jour, il se produit dans nos programmes une somme faramineuse d'informations cliniques qui aussitôt dispensée tombe aux oubliettes. Pensez à toutes les présentations que vous avez dû donner... Et si ces producteurs de contenu se fédéraient afin de ne plus travailler en isolation et unissaient leurs efforts. Et si tout ce travail à titre d'étudiant ou de clinicien pouvait bénéficier d'un deuxième souffle et servir de fondation ou d'amélioration d'une base de connaissances médicales de qualité, impartiale, à jour, collaborative et en libre accès. Voilà justement le principal objectif que Wikimedica s'est donné (http://wikimedi.ca) (Figure 1, Figure 2). Ça, mais aussi de rassembler sur une plateforme tous les professionnels de la santé afin de briser les cloisons historiques qui séparent chaque spécialité : la collaboration interprofessionnelle est,

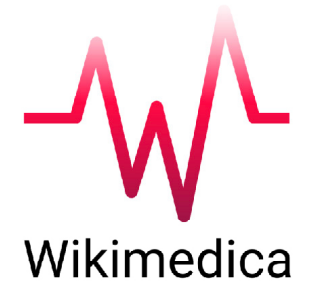

Figure 1. Le logo de Wikimedica (http://wikimedi.ca)

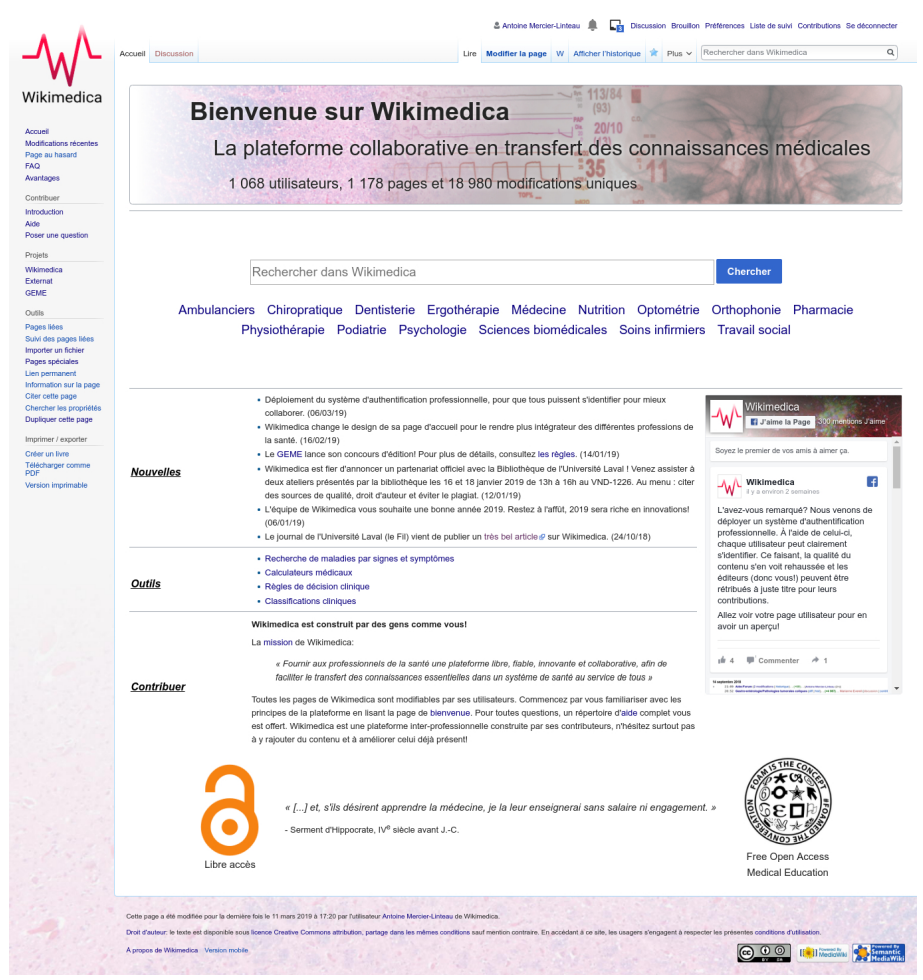

Figure 2. La page d'accueil intègre des liens vers les portails de chaque profession, des nouvelles et une barre de recherche permettant de naviguer tout le contenu de la plateforme. après tout, plus que jamais actuelle. Et qui de mieux pour enseigner l'examen de l'épaule qu'un physiothérapeute?

Pour ce faire, le format du wiki s'est imposé comme le plus adapté: une plateforme où les utilisateurs sont aussi éditeurs et appelés à bonifier le contenu dans le cadre d'un processus éditorial révisé par les pairs. Peuvent se retrouver surWikimedica une multitude d'outils: résumés cliniques, algorithmes, protocoles, médicaments, outils pédagogiques (cours, flashcards, cas cliniques, etc.), calculateurs médicaux, etc. En fait, il n'y a pas de limite à ce qui peut être intégré à Wikimedica et dans ce cadre, le projet vise à contrer la multiplication des sources d'informations cliniques (applications, PDF, documents papiers...) en offrant une consultation efficace et efficiente, que ce soit dans le cadre d'un travail scolaire ou au chevet d'un patient. À cet effet, une ontologie a été développée afin de standardiser la conception des pages. Plusieurs mécanismes ont également été mis en place pour éviter la péremption de l'information par sa duplication. Différentes pages partageant un même contenu peuvent donc se parler pour mutuellement se tenir à jour lorsque l'une est modifiée. Par ailleurs, la connaissance sur Wikimedica est structurée de deux façons simultanées : une page définit du texte lisible par I'humain, mais aussi des données sémantiques navigables par un programme informatique. Par exemple, il vous est possible de demander au système de vous indiquer toutes les maladies dont la dyspnée est un symptôme. En étoffant un peu votre requête avec d'autres symptômes et des signes, vous obtiendrez un diagnostic différentiel pour une présentation clinique donnée. Les possibilités sont donc infinies, surtout lorsque l'on met ces données en relation avec d'autres. En association avec un dossier médical électronique, la révision de la qualité de l'acte pourrait devenir automatisée. À plus grande échelle et avec le concours de l'intelligence artificielle, Wikimedica pourrait s'intégrer à un assistant de prise en charge clinique, personnalisant ses recommandations selon le patient. Les intelligences artificielles (IA), qui ont déjà fait leur entrée en santé, méritent que l'on s'y attarde. La preuve sera bientôt faite qu'en les associant à un clinicien, elles amélioreront l'issue des soins à un patient, les rendant ainsi indispensables (4). Imaginez donc le préjudice que subiraient nos systèmes de santé publics s'il fallait que ces IA soient contrôlées par des intérêts mercantiles et alimentées par de la donnée fermée. Et contrairement au scénario UpToDate discuté en début de texte, passer au compétiteur sera loin d'être une mince affaire. Maintenant, imaginez l'ampleur du problème éthique si nous n'avions aucun moyen de savoir 
si les recommandations d'un tel outil sont motivées par un partenariat entre le fournisseur et un tiers ou le cheminement logique ayant mené un algorithme à proposer un traitement s'étant avéré préjudiciable (5). Ici, Wikimedica se positionnerait comme source de données libre et ouvertes, s'alliant au privé pour fournir des systèmes d'IA transparents et non-exclusifs.

Wikimedica partage les mêmes idéaux fondateurs que Wikipédia : la libération de la connaissance, la collaboration wiki comme mode éditorial et la même plateforme technique (MediaWiki). Cependant, il existe trois différences clés sur Wikimedica:

1. Il est destiné aux professionnels de la santé;

2. Seuls les professionnels de la santé peuvent le modifier;

3. L'anonymat n'y est pas permis.

Ces contraintes vont de soi. Wikipédia est avant tout grand public, alors que Wikimedica contient de l'information technique. Conséquemment, Wikimedica ne peut être modifié que par des professionnels (ou étudiants de ces professions) et ces derniers ne peuvent être anonymes, car ils doivent engager leur responsabilité déontologique, garantissant ainsi la qualité de leurs contribution stout en permettant la reconnaissance de leurs apports. Le processus éditorial n'appartient donc plus à un petit noyau, mais à la communauté entière et est mis en place par tout un système de suivis des modifications, d'alertes, de discussion et de suggestions. II ne se change pas une virgule sur Wikimedica sans que l'on sache qui l'a fait, où et à quel moment. La connaissance sensible, par exemple un dosage, y est protégée, la fluidité du processus éditorial fait en sorte qu'une information erronée peut être corrigée instantanément et les dernières avancées sont intégrées sans délai.

Avec Wikimedica, le transfert des connaissances devient dynamique et collaboratif. II reste que cette manière de faire constitue un changement de paradigme et beaucoup voient d'un mauvais œil la possibilité que leur travail puisse être modifié par d'autres. Pourtant, l'expérience nous démontre que les contributions sur des systèmes collaboratifs vont dans le sens de l'amélioration (6). Pour une plateforme sans anonymat telle que Wikimedica, le risque de vandalisme est virtuellement nul, surtout lorsqu'on le compare aux bénéfices d'un cycle d'améliorations raccourci et collaboratif. Également, d'autres ont tendance à être suspicieux du fait que l'accès en lecture soit public. Wikimedica n'a cependant pas inventé Dr. Google et ne croit pas non plus que l'information devrait être censurée dans la seule crainte qu'elle soit mal utilisée: faisons confiance au jugement citoyen. Nos sociétés fourmillent d'esprits créatifs, nul besoin d'être médecin pour innover en médecine. II y a donc fort à parier qu'un individu inventif trouvera des façons de repenser la connaissance diffusée par un système en libre accès pour solutionner d'autres défis. C'est dans ce contexte que le libre accès prend toute son importance et vient agir comme catalyseur de l'innovation. Pourtant, lorsque présenté aux décideurs, le concept accroche, possiblement puisque les licences libre accès sont perçues à tort comme une cession de droits et une perte de contrôle. En fait, en plus d'encourager le progrès, le libre accès donne une portée bien plus grande aux œuvres et s'impose comme un impératif éthique, comme l'a formulé I'UNESCO en 1999 (7):

"L'égalité d'accès à la science ne répond pas seulement à un impératif social et éthique du développement humain, elle est aussi indispensable si l'on veut exploiter pleinement le potentiel des communautés scientifiques dans le monde entier et faire tendre le progrès scientifique vers la satisfaction des besoins de l'humanité (8). »

Disons que le libre accès a le vent dans les voiles. Le journal même dans lequel vous lisez ces lignes en est la preuve, mais aussi le fait que ce mode de publication ait été adopté pas des grands noms de l'éducation comme le MIT ou Johns Hopkins, sans oublier qu'à partir de 2020, toute recherche financée par I'Union Européenne devra être publiée en libre accès $(9,10)$.

Au moment de la rédaction de ce texte et après deux ans d'existence portée par ses bénévoles, Wikimedica compte 1 068 utilisateurs (plus de 95\% des étudiants en médecine de I'Université Laval), 1172 pages, une bonne partie des notes de cours du préclinique et 18954 modifications. La plateforme vient de s'ouvrir aux autres universités et autres programmes de la santé. Dans un futur proche, en plus de l'expansion du contenu général, Wikimedica se donne comme objectif de créer un corpus de pages couvrant les sujets du EACMC partie 1, de poursuivre le développement de ses partenariats, de permettre la reconnaissance de l'édition comme formation continue, de se déployer dans des pays en voie de développement... et bien d'autres.

Dans l'immédiat, Wikimedica n'est disponible qu'en français mais ce n'est pas parce que le concept ne s'appliquerait pas au monde anglo-saxon, bien au contraire. Le choix initial de langue provient du fait que l'université dans laquelle le projet a pris forme est francophone et qu'une initiative de ce type a plus de chances de prendre racine dans cette communauté relativement homogène du monde médical nord-américain. 
L'avènement d'une version anglaise de Wikimedica n'est donc qu'une question de temps. Autrement, Wikimedica est ouvert aux contributeurs de tous horizons, tant ceux intéressés à améliorer la qualité de l'information, à rédiger du contenu ou encore à porter le projet ainsi que ses principes dans leur communauté respective, qu'elle soit étudiante ou professionnelle. Le projet n'a pas comme ambition de convaincre les professionnels en pratique de changer leurs habitudes, mais tentera de prendre racine dans la relève médicale issue de la génération du web et le monde de l'éducation, milieux chez qui les idéaux de libre accès et de responsabilité sociale portés par Wikimedica résonneront tout particulièrement. Sur ce, je vous laisse avec notre énoncé de mission :

«Fournir aux professionnels de la santé une plateforme libre, fiable, innovante et collaborative, afin de faciliter le transfert des connaissances essentielles dans un système de santé au service de tous (11). »

\section{RÉFÉRENCES}

1. Dynamed remplace UpToDate [Internet]. Université de Sherbrooke: Faculté de médecine et des sciences de la santé; 29 mai 2014 [cité le 24 mars 2019]. Disponible à https://www.usherbrooke.ca/medecine/accueil/babillard-interne/babillard-details/article/25598/

2. Allahwala UK, Nadkarni A, Sebaratnam DF. Wikipedia use amongst medical students-new insights into the digital revolution. Medical teacher 2013; 35 (4): 337

3. Heilman J, Open Access to a High-Quality, Impartial, Point-of-Care Medical Summary Would Save Lives: Why Does It Not Exist? PLoS Med [Internet]. 25 août 2015 [cité le 24 mars 2019]. Disponible à: https://doi.org/10.1371/ journal.pmed.1001868

4. Haenssle H, Fink C, Schneiderbauer R, et al. Man against machine: diagnostic performance of a deep learning convolutional neural network for dermoscopic melanoma recognition in comparison to 58 dermatologists. Annals of Oncology 2018; 29 (8): 1836-1842

5. Rigby M. Ethical Dimensions of Using Artificial Intelligence in Health Care [Internet]. AMA Journal of Ethics; février 2019 [cité le 19 avril 2019]. Disponible à: https://journalofethics.ama-assn.org/article/ethical-dimensions-using-artificial-intelligence-health-care/2019-02

6. Azzam A, Bresler D, Leon A et Maggio L. Why Medical Schools Should Embrace Wikipedia. Academic Medicine (2017); 92 (2) : 194-200

7. Li Y, Wu C, Yan E, Li K Will open access increase journal CiteScores? An empirical investigation over multiple disciplines. PLoS ONE [Internet]. 30 août 2018 [cité le 24 mars 2019]. Disponible à: https://doi.org/10.1371/journal. pone. 0201885

8. Politique de libre accès aux publications de I'UNESCO [Internet]: UNESCO; [cité le 24 mars 2019]. Disponible à: http://www.unesco.org/new/fileadmin/MULTIMEDIA/HQ/ERI/pdf/oa_policy_fr.pdf

9. What is open access? [Internet] Johns Hopkins, Office of the Provost [cité le 19 avril 2019]. Disponible à: https://provost.jhu.edu/about/open-access/about/ 